\title{
Oscillating global continua of positive solutions of second order Neumann problem with a set- valued term
}

Dongming Yan

Correspondence: yandong_ming@126.com Department of Mathematics, Sichuan University, Chengdu 610064, China

\author{
Abstract \\ In this note, we study the oscillating global continua of the differential inclusion of \\ the form

$$
\left\{\begin{array}{l}
-u^{\prime \prime}+q u \in \lambda F(\cdot, u) \\
u^{\prime}(0)=0, \quad u^{\prime}(1)=0
\end{array}\right.
$$ \\ where $F$ is a "set-valued representation" of a function with jump discontinuities along \\ the line segment $[0,1] \times\{0\}$, and $\lambda \in[0, \infty)$ is a parameter. The proof of our main \\ result relies on an approximation procedure. \\ Mathematics Subject Classification 2000: 34B16; 34B18.
}

Keywords: climate model, differential inclusion, eigenvalue, positive solutions

\section{Introduction}

In recent years, nonsmooth analysis has come to play an important role in functional analysis [1], dynamical systems [2], control theory [3], optimization [4], mechanical systems [5], differential equation [6,7] etc. Since many mathematical and physical problems may be reduced to ODES or PDES with discontinuous nonlinearities, the existence of multiple solutions for differential inclusion problems has been widely investigated [8-19].

In this article, we are concerned with the following differential inclusion problem which raises from a Budyko-North type energy balance climate models:

$$
\left\{\begin{array}{l}
-u^{\prime \prime}(x)+q(x) u(x) \in \lambda F(x, u(x)), \quad \text { a.e. } x \in(0,1) \\
u^{\prime}(0)=0, \quad u^{\prime}(1)=0
\end{array}\right.
$$

(see [20-25] and the references therein). In particular, the set-valued right hand side arise from a jump discontinuity of the albedo at the ice-edge in these models. By filling such a gap, one arrives at the set-valued problem (1.1). As in [25], we are here interested in a considerably simplify version as compared to the situation from climate modeling, e.g. a one-dimensional regular Sturm-Liouville differential operator substitutes for a two-dimensional Laplace-Beltrami operator or a singular Legendre-type operator, and the jump discontinuity is transformed to $u=0$ in a way, which resembles only locally the climatological problem. 
We are concerned with the set-valued problem (1.1) under the following assumptions

(H1) $q \in C([0,1],(0,+\infty))$;

$\left.\left.\begin{array}{llllll}(\mathrm{H} 2) & f^{+} \in & C & ([0, & 1\end{array}\right] \quad \times \quad[0,+\infty), \quad(0,+\infty)\right)$,

$\inf _{x \in[0,1]} f^{+}(x, 0)>0, \quad \lim _{s \rightarrow+\infty} \frac{f^{+}(x, s)}{s}=b(x) \in C([0,1],(0, \infty))$.

Let the set-valued function $F$ in (1.1) is given by

$$
F(x, y)=\left\{\begin{array}{l}
\left\{f^{+}(x, y)\right\}, \quad x \in[0,1], \quad y>0, \\
{\left[0, f^{+}(x, 0)\right], \quad x \in[0,1] .}
\end{array}\right.
$$

Notice that if $f^{+}(x, 0) \equiv 0, x \in[0,1]$, then the differential inclusion problem (1.1) reduces to the BVP of differential equation

$$
\left\{\begin{array}{l}
-u^{\prime \prime}(x)+q(x) u(x)=\lambda f^{+}(x, u(x)), \quad x \in(0,1) \\
u^{\prime}(0)=0, \quad u^{\prime}(1)=0 .
\end{array}\right.
$$

In the last 20 years, the positive solutions of (1.2) have been studied by several authors, see Jiang and Liu [26], Chu et al. [27] and Sun et al. [28].

The purpose of this article is to investigate the oscillating global continua of positive solutions of the differential inclusion problem (1.1). The proof of our main result relies on an approximation procedure. The rest of the article is organized as follows. In Section 2, we state some notations and prove some preliminary results. In Section 3, we state and prove our main result. In Section 4, an example is given to illustrate the application of our main result.

\section{Notations and preliminaries}

Recall Kuratowski's notion of lower and upper limits of sequence of sets.

Definition 2.1. [29] Let $X$ be a metric space and $\left\{Z_{l}\right\}_{l \in \mathbb{N}}$ be a sequence of subsets of $X$. The set

$$
\limsup _{l \rightarrow \infty} Z_{l}:=\left\{x \in X: \liminf _{l \rightarrow \infty} \operatorname{dist}\left(x, Z_{l}\right)=0\right\}
$$

is called the upper limit of the sequence $\left\{Z_{l}\right\}$, whereas

$$
\liminf _{l \rightarrow \infty} Z_{l}:=\left\{x \in X: \lim _{l \rightarrow \infty} \operatorname{dist}\left(x, Z_{l}\right)=0\right\}
$$

is called the lower limit of the sequence $\left\{Z_{l}\right\}$.

Definition 2.2. [29] A component of a set $M$ is meant a maximal connected subset of M.

Lemma 2.1. [29] Suppose that $Y$ is a compact metric space, $A$ and $B$ are non-intersecting closed subsets of $Y$, and no component of $Y$ intersects both $A$ and $B$. Then there exist two disjoint compact subsets $Y_{A}$ and $Y_{B}$, such that $Y=Y_{A} \cup Y_{B}, A \subset Y_{A}, B \subset Y_{B}$.

Using the above Whyburn Lemma, Ma and An [30] proved the following

Lemma 2.2. [30, Lemma 2.1] Let $Z$ be a Banach space and let $\left\{A_{n}\right\}$ be a family of closed connected subsets of $Z$. Assume that

(i) there exist $z_{n} \in A_{n}, n=1,2, \ldots$, and $z^{*} \in Z$, such that $z_{n} \rightarrow z^{*}$;

(ii) $r_{n}=\sup \left\{\|x\| \mid x \in A_{n}\right\}=\infty$; 
(iii) for every $R>0$, $\left(\bigcup_{n=1}^{\infty} A_{n}\right) \cap B_{R}$ is a relatively compact set of $Z$, where $B_{R}=\{x \in Z$ $\mid\|x\| \leq R\}$. Then there exists an unbounded component $\mathcal{C}$ in $\limsup _{l \rightarrow \infty} A_{l}$ and $z^{*} \in \mathcal{C}$.

Remark 2.1. The limiting processes for sets go back at least to the work of Kuratowski [31]. Lemma 2.2 is a slight generalization of the following well-know result due to Whyburn [29]:

Proposition 2.1. (Whyburn [29, p. 12]) Let $Z$ be a Banach space and $\left\{A_{n}\right\}$ be a family of closed connected subsets of $Z$. Let $\liminf _{l \rightarrow \infty} A_{l} \neq \emptyset$ and $\cup_{l \in \mathbb{N}} A_{l}$ is relatively compact. Then $\limsup _{l \rightarrow \infty} A_{l}$ is nonempty, compact and connected.

Next, we introduce the result of global solution behavior of the bifurcation branches of the equation

$$
x=\mu(L x+N x), \quad \mu \in R, \quad x \in X,
$$

to wit the following lemma.

Lemma 2.3. [32] (Dancer (1974)) Assume that

(C1) The operators $L, N: X \rightarrow X$ are compact on the Banach space $X$ over $R$. Furthermore, $L$ is linear and $\|N x\| /\|x\| \rightarrow 0$ as $\|x\| \rightarrow 0$;

(C2) The real number $\mu_{0}$ is a characteristic number of $L$ of odd algebraic multiplicity;

$(C 1+)$ The real Banach space $X$ has an order cone $K$ with $X=K-K$, i.e., every $x \in X$ can be represented as $x=x_{1}-x_{2}$, where $x_{1}, x_{2} \in K$. Furthermore, $L+N$ is positive, i.e., $L+N$ maps $K$ into $K$;

$(C 2+)$ The spectral radius $r(L)$ of $L$ is positive. We set $\mu_{0}=(r(L))^{-1}$.

Then $\left(\mu_{0}, 0\right)$ is a bifurcation point of equation (2.1) and

$$
S_{+}:=\overline{\{(\mu, x) \in R \times X:(\mu, x) \text { is a solution of }(2.1) \text { with } \mu>0, x>0\}}
$$

contains an unbounded solution component $C_{1}^{+}$which passes through $\left(\mu_{0}, \boldsymbol{0}\right)$.

If additionally

(C3+) The linear operator $L$ is strongly positive, then $(\mu, x) \in C_{1}^{+}$and $\mu \neq \mu_{0}$ always implies $x>0$ and $\mu>0$.

Remark 2.2. This result is often called the nonlinear Krein-Rutman theorem. It will play an important role in the proof of our main result.

Let $\phi$ and $\psi$ be the unique solution of the problems

$$
\left\{\begin{array}{l}
-u^{\prime \prime}(x)+q(x) u(x)=0, \quad x \in(0,1) \\
u^{\prime}(0)=0, \quad u(0)=1
\end{array}\right.
$$

and

$$
\left\{\begin{array}{l}
-u^{\prime \prime}(x)+q(x) u(x)=0, \quad x \in(0,1) \\
u^{\prime}(1)=0, \quad u(1)=1
\end{array}\right.
$$

respectively. Then it is easy to check $\phi(\cdot)$ is nondecreasing on $(0,1), \psi(\cdot)$ is nonincreasing on $(0,1)$, and the Green's function $G(x, s)$ of

$$
\left\{\begin{array}{l}
-u^{\prime \prime}(x)+q(x) u(x)=0, \quad x \in(0,1) \\
u^{\prime}(0)=0, \quad u^{\prime}(1)=0
\end{array}\right.
$$


is explicitly given by

$$
G(x, s)=-\frac{1}{\psi^{\prime}(0)}\left\{\begin{array}{l}
\psi(x) \varphi(s), 0 \leq s \leq x \leq 1 \\
\varphi(x) \psi(s), 0 \leq x \leq s \leq 1
\end{array}\right.
$$

Moreover, we have that

$$
\begin{aligned}
& 0<G(x, s) \leq G(s, s),(x, s) \in[0,1] \times[0,1] \\
& \sigma G(s, s) \leq G(x, s), \quad(x, s) \in[0,1] \times[0,1] \\
& \text { with } \sigma:=\min \left\{\frac{1}{\psi(0)}, \frac{1}{\varphi(1)}\right\} .
\end{aligned}
$$

\section{The main result}

Let $\Sigma$ be the closure of the set of positive solutions of $(1.1)$ in $[0, \infty) \times C^{1}[0,1]$, and $\mathbb{N}^{*}$ $:=\{1,2, \ldots, N\}$. The main result of this article is the following theorem.

Theorem 3.1. Assume that (H1)-(H2) hold. If

(H3) there is an increasing sequence of positive numbers $\left\{\xi_{j}\right\}_{1}^{N}$ and a small enough constant $\delta$ such that $\xi_{1}<\sigma\left(\xi_{2}-\delta\right)$ and

$$
\begin{gathered}
\Phi\left(\xi_{2 j-1}\right)<\frac{1}{2}\left(\int_{0}^{1} G(s, s) d s\right)^{-1}\left(\xi_{2 j-1}-\delta\right), \quad j \in \mathbb{N}^{*} \\
\Psi\left(\xi_{2 j}\right)>2\left(\int_{0}^{1} G\left(\frac{1}{2}, s\right) d s\right)^{-1}\left(\xi_{2 j}+\delta\right), \quad j \in \mathbb{N}^{*}
\end{gathered}
$$

where

$$
\begin{gathered}
\Phi(l):=\max \left\{f^{+}(t, c): 0 \leq t \leq 1, \quad 0 \leq c \leq l+\delta\right\} \\
\Psi(l):=\min \left\{f^{+}(t, c): 0 \leq t \leq 1, \quad \sigma(l-\delta) \leq c \leq l+\delta\right\}
\end{gathered}
$$

then there exits an unbounded component $C_{1}^{+}$in $\Sigma$ with $(0,0) \in C_{1}^{+}$. Moreover,

(i) $(\lambda, u) \in C_{1}^{+}$with $\|u\|_{\infty}=\xi_{2 j-1}$ for some $j \in \mathbb{N}^{*}$ implies that $\lambda \geq 2$;

(ii) $(\lambda, u) \in C_{1}^{+}$with $\|u\|_{\infty}=\xi_{2 j}$ for some $j \in \mathbb{N} *$ implies that $\lambda \leq \frac{1}{2}$.

Actually, such continua $C_{1}^{+}$can be obtained as upper limits in the sense of Kuratowski of sequence of solution continua from associated continuous problems. To this end one sets

$$
d_{g}:=\inf \left\{f^{+}(x, 0): x \in[0,1]\right\}
$$

fixes $l_{0} \in \mathbb{N}$ such that $\frac{d_{g}}{l_{0}}<\xi_{1}$, and selects an approximation sequence $\left\{f_{l}\right\} \subset C([0$, $1] \times \mathbb{R}, \mathbb{R})\left(l>l_{0}\right)$ of $F$ satisfying:
(A1) $f_{l}(x, y)=l y$ for $x \in[0,1]$ and $y \in\left[0, \frac{d_{g}}{2 l}\right]$;
(A2) $\frac{d_{g}}{2} \leq f_{l}(x, y) \leq f^{+}(x, y)$ for $x \in[0,1]$ and $y \in\left[\frac{d_{g}}{2 l}, \frac{d_{g}}{l}\right]$; 
(A3) $f_{l}(x, y)=f^{+}(x, y)$ for $x \in[0,1]$ and $y \geq \frac{d_{g}}{l}$;

(A4) $\left\{f_{l}(x, y)\right\}_{l \in \mathbb{N}}$ is nondecreasing in $l$ for $(x, y) \in[0,1] \times(0, \infty)$.

Next, we show that the continuous problem

$$
\left\{\begin{array}{l}
-v^{\prime \prime}(x)+q(x) v(x)=\lambda f_{l}(x, v(x)), \quad x \in(0,1) \\
v^{\prime}(0)=0, \quad v^{\prime}(1)=0
\end{array}\right.
$$

has an unbounded closed subsets $C_{1, l}^{+}$of the positive solutions set of $\left(3.2_{l}\right)$ with

(a) $\left(\frac{\lambda_{1}}{l}, 0\right)$ is the bifurcation point contained in $C_{1, l}^{+}$;

(b) If $(\mu, \vartheta) \in C_{1, l}^{+}$and $\vartheta \otimes 0$, then $\vartheta$ is positive on $(0,1)$.

It is easy to see that $\left(3.2_{l}\right)$ equivalent to

$$
v(x)=\lambda \int_{0}^{1} G(x, s) f_{l}(s, v(s)) \mathrm{d} s .
$$

Let

$$
\begin{aligned}
& (L v)(x):=l \int_{0}^{1} G(x, s) v(s) \mathrm{d} s \\
& (N v)(x):=\int_{0}^{1} G(x, s)\left(f_{l}(s, v(s))-l v(s)\right) \mathrm{d} s, \quad v \in C[0,1] .
\end{aligned}
$$

Then according to (3.3), $\left(3.2_{l}\right)$ can be written as the following operator equation

$$
v=\lambda(L v+N v) \text {. }
$$

Clearly, the operators $L, N: C[0,1] \rightarrow C[0,1]$ are compact on the Banach space $C[0,1]$. Furthermore, $L$ is linear and thanks to (2.3)(A1) that

$$
\begin{aligned}
\frac{\|N v\|_{\infty}}{\|v\|_{\infty}} & =\left\|\int_{0}^{1} G(x, s) \frac{f_{l}(s, v(s))-l v(s)}{\|v\|_{\infty}} \mathrm{d} s\right\|_{\infty} \\
& \leq \int_{0}^{1} G(s, s) \frac{\left|f_{l}(s, v(s))-l v(s)\right|}{\|v\|_{\infty}} \mathrm{d} s \rightarrow 0, \quad \text { as }\|v\|_{\infty} \rightarrow 0,
\end{aligned}
$$

which implies that the condition (C1) of Lemma 2.3 is satisfied.

Denote the principal eigenvalue of

$$
\left\{\begin{array}{l}
-\omega^{\prime \prime}(x)+q(x) \omega(x)=\lambda \omega(x), \quad x \in(0,1) \\
\omega^{\prime}(0)=0, \quad \omega^{\prime}(1)=0
\end{array}\right.
$$

by $\lambda_{1}$, then we know that $\lambda_{1}>0$ (see [33]). Since (3.4) is equivalent to operator equation

$$
\omega=\frac{\lambda}{l} L \omega,
$$


we have that $(r(L))^{-1}=\frac{\lambda_{1}}{l}$. Therefore, the conditions $(\mathrm{C} 2)(\mathrm{C} 2+)$ of Lemma 2.3 are satisfied.

Let the cone $K$ in $C[0,1]$ is given by

$$
K=\{u \in C[0,1] \mid u(x) \geq 0,0 \leq x \leq 1\} .
$$

It is easy to see thanks to (A1)-(A4) and $(2.3)$ that the $(\mathrm{C} 1+)(\mathrm{C} 3+)$ conditions of Lemma 2.3 are satisfied.

According to Lemma 2.3, we obtain that $\left(\frac{\lambda_{1}}{l}, 0\right)$ is a bifurcation point of the positive solutions set of $\left(3.2_{l}\right)$ for every $l \in\left\{l_{0}+1, l_{0}+2, \ldots\right\}=: \mathbb{N}_{0}$, and for each $l \in \mathbb{N}_{0}$ there exits an unbounded closed subsets $C_{1, l}^{+}$of the positive solutions set of $\left(3.2_{l}\right)$ with (a) and (b).

Combining the above with the fact

$$
\lim _{l \rightarrow \infty}\left(\frac{\lambda_{1}}{l}, 0\right)=(0,0)
$$

and utilizing Lemma 2.2, it concludes that there exits an unbounded component $C_{1}^{+}$ with

$$
(0,0) \in C_{1}^{+}
$$

and

$$
C_{1}^{+} \subset \limsup _{l \rightarrow \infty} C_{1, l}^{+} .
$$

Denote the cone $P$ in $C[0,1]$ by

$$
P=\left\{u \in C[0,1] \mid \min _{0 \leq x \leq 1} u(x) \geq \sigma\|u\|_{\infty}\right\} .
$$

Define an operator $T_{\lambda}: P \rightarrow C[0,1]$ by

$$
\left(T_{\lambda} u\right)(x)=\lambda \int_{0}^{1} G(x, s) f_{l}(s, u(s)) \mathrm{d} s, \quad x \in[0,1] .
$$

It is easy to get the following lemma.

Lemma 3.1. Assume that (H1), (H2) and (A1)-(A4) hold. Then $T_{\lambda}: P \rightarrow P$ is completely continuous.

Lemma 3.2. Assume that (H1), (H2) and (A1)-(A4) hold. If $0 \leq u(x) \leq r, r>0$, for $x \in$ $[0,1]$, then

$$
\left\|T_{\lambda} u\right\|_{\infty} \leq \lambda M_{r} \int_{0}^{1} G(s, s) d s,
$$

where $M_{r}=\max _{0 \leq x \leq 1,0 \leq s \leq r}\left\{f_{l}(x, s)\right\}$. 
Proof. Since $f_{l}(x, u(x)) \leq M_{r}$ for $x \in[0,1]$, it follows from (2.3) that

$$
\begin{aligned}
\left\|T_{\lambda} u\right\|_{\infty} & =\left\|\lambda \int_{0}^{1} G(x, s) f_{l}(s, u(s)) \mathrm{d} s\right\|_{\infty} \\
& \leq \lambda \int_{0}^{1} G(s, s) f_{l}(s, u(s)) \mathrm{d} s \\
& \leq \lambda M_{r} \int_{0}^{1} G(s, s) \mathrm{d} s .
\end{aligned}
$$

Lemma 3.3. Assume that (H1), (H2) and (A1)-(A4) hold. If $\sigma(r-\delta) \leq u(x) \leq r+\delta, r$ $>\delta$, for $x \in[0,1]$, then

$$
\left\|T_{\lambda} u\right\|_{\infty} \geq \lambda m_{r} \int_{0}^{1} G\left(\frac{1}{2}, s\right) d s,
$$

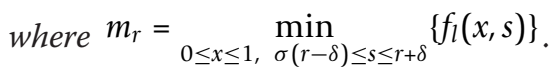

Proof. Since $f_{l}(x, u(x)) \geq m_{r}$ for $x \in[0,1]$, it follows that

$$
\begin{aligned}
\left\|T_{\lambda} u\right\|_{\infty} & \geq \lambda \int_{0}^{1} G\left(\frac{1}{2}, s\right) f_{l}(s, u(s)) \mathrm{d} s \\
& \geq \lambda m_{r} \int_{0}^{1} G\left(\frac{1}{2}, s\right) \mathrm{d} s .
\end{aligned}
$$

Lemma 3.4. Assume that (H1), (H2), (H3) and (A1)-(A4) hold. then

(i) $(\lambda, u) \in C_{1, l}^{+}$with $\|u\|_{\infty} \in\left(\xi_{2 j-1}-\delta, \xi_{2 j-1}+\delta\right)$ for some $j \in \mathbb{N} *$ implies that $\lambda>2$;

(ii) $(\lambda, u) \in C_{1, l}^{+}$, with $\|u\|_{\infty} \in\left(\xi_{2 j}-\delta, \xi_{2 j}+\delta\right)$ for some $j \in \mathbb{N}^{*}$ implies that $\lambda<\frac{1}{2}$.

Proof. (i) Assume that $(\lambda, u) \in C_{1, l}^{+}$with $\|u\|_{\infty} \in\left(\xi_{2 j-1}-\delta\right.$, $\left.\xi_{2 j-1}+\delta\right)$ for some $j \in \mathbb{N}^{*}$, then $u=T_{\lambda} u$ and

$$
0 \leq u(x) \leq \xi_{2 j-1}+\delta \quad \text { for } x \in[0,1] .
$$

By Lemma 3.2 and (H3), it follows that

$$
\begin{aligned}
\|u\|_{\infty} & =\left\|T_{\lambda} u\right\|_{\infty} \\
& \leq \lambda M_{\xi_{2 j-1}+\delta} \int_{0}^{1} G(s, s) \mathrm{d} s \\
& =\lambda \max _{0 \leq x \leq 1,0 \leq s \leq \xi_{2 j-1}+\delta}\left\{f_{l}(x, s)\right\} \int_{0}^{1} G(s, s) \mathrm{d} s \\
& \leq \lambda \Phi\left(\xi_{2 j-1}\right) \int_{0}^{1} G(s, s) \mathrm{d} s \\
& <\lambda\left[\frac{1}{2}\left(\int_{0}^{1} G(s, s) \mathrm{d} s\right)^{-1}\left(\xi_{2 j-1}-\delta\right)\right] \int_{0}^{1} G(s, s) \mathrm{d} s . \\
& =\frac{1}{2} \lambda\left(\xi_{2 j-1}-\delta\right) .
\end{aligned}
$$


Thus $\lambda>2$.

(ii) Assume that $(\lambda, u) \in C_{1, l}^{+}$with $\|u\|_{\infty} \in\left(\xi_{2 j}-\delta\right.$, $\left.\xi_{2 j}+\delta\right)$ for some $j \in \mathbb{N}^{*}$, then $u=$ $T_{\lambda} u$ and

$$
\sigma\left(\xi_{2 j}-\delta\right) \leq u(x) \leq \xi_{2 j}+\delta \quad \text { for } x \in[0,1] .
$$

By Lemma 3.3 and the assumption (H3), it follows that

$$
\begin{aligned}
\|u\|_{\infty} & =\left\|T_{\lambda} u\right\|_{\infty} \\
& \geq \lambda m_{\xi_{2 j}} \int_{0}^{1} G\left(\frac{1}{2}, s\right) \mathrm{d} s \\
& =\lambda \operatorname{lox\leq 1,}_{\sigma\left(\xi_{2 j}-\delta\right) \leq s \leq \xi_{2 j}+\delta}\left\{f_{l}(x, s)\right\} \int_{0}^{1} G\left(\frac{1}{2}, s\right) \mathrm{d} s \\
& =\lambda \Psi\left(\xi_{2 j}\right) \int_{0}^{1} G\left(\frac{1}{2}, s\right) \mathrm{d} s \\
& >\lambda\left[2\left(\int_{0}^{1} G\left(\frac{1}{2}, s\right) \mathrm{d} s\right)^{-1}\left(\xi_{2 j}+\delta\right)\right] \int_{0}^{1} G\left(\frac{1}{2}, s\right) \mathrm{d} s . \\
& =2 \lambda\left(\xi_{2 j}+\delta\right) .
\end{aligned}
$$

Thus $\lambda<\frac{1}{2}$.

Lemma 3.5. If $(\lambda, u) \in C_{1}^{+}$, then $(\lambda, u)$ is a solution of $(1.1)$ and $u \in W^{2, \infty}(0,1)$.

Proof. Let $(\lambda, u) \in C_{1}^{+}$. By the definition of $C_{1}^{+}$there exists a sequence $\left\{l_{k}\right\} \in \mathbb{N}_{0}$ strictly increasing, and $\left(\lambda_{l_{k}}, v_{l_{k}}\right) \in[0, \infty) \times C^{1}[0,1]$ with $\left(\lambda_{l_{k}}, v_{l_{k}}\right) \in C_{1, l_{k}}^{+}$for $k \in \mathbb{N}$ and

$$
\left(\lambda l_{l_{k}}, v_{l_{k}}\right) \rightarrow(\lambda, u) .
$$

Since $\left\{f_{l_{k}}\left(\cdot, v_{l_{k}}(\cdot)\right)\right\}$ is uniformly bounded, i.e.

$$
\left\|f_{l_{k}}\right\|_{L^{2}} \leq M
$$

we can assume after passing to a subsequence, if necessary, that it converges weekly in $L^{2}(0,1)$ to some $\varphi$. We claim that $\varphi(x) \in F(x, u(x))$ a.e. on $(0,1)$.

Let $x_{0} \in(0,1)$ with $u\left(x_{0}\right)>0$. Then there exist $\rho>0$ and $\tau \in\left(0, \min \left\{x_{0}, 1-x_{0}\right\}\right)$ with $u(x)>\rho$ for all $x \in\left(x_{0}-\tau, x_{0}+\tau\right)$, hence there is a $k_{0} \in \mathbb{N}$ with $v_{l_{k}}(x)>\frac{\rho}{2}$ for all $k>k_{0}$ and $x \in\left(x_{0}-\tau, x_{0}+\tau\right)$. Choose $k_{1}>k_{0}$ with $\frac{d_{g}}{l_{k_{1}}}<\frac{\rho}{2}$. Then $f_{l_{k}}\left(x, v_{l_{k}}(x)\right)=f^{+}\left(x, v_{l_{k}}(x)\right)$ for all $k \geq k_{1}$ and $x \in\left(x_{0}-\tau, x_{0}+\tau\right)$, which yields $\varphi(x)=f^{+}(x, u(x))$ for $x \in\left(x_{0}-\tau, x_{0}\right.$ $+\tau)$ a.e.

Next, if $u \equiv 0$, let $K:=\left\{x \in(0,1): \varphi(x)>f^{+}(x, 0)\right\}$. We claim that meas $(K)=0$. Suppose that meas $(K)>0$. Then $\varepsilon:=\int_{K}\left[\varphi(x)-f^{+}(x, 0)\right] \mathrm{d} x>0$, and one finds $\eta \in(0, \infty)$ with meas $(K)\left|f^{+}(x, y)-f^{+}(x, 0)\right| \leq \frac{\varepsilon}{2}$ for $x \in[0,1]$ and $y \in[0, \eta]$. Choosing $k_{2} \in \mathbb{N}$ with $\left\|v_{l_{k}}-u\right\|_{\infty}<\eta$ for $k \geq k_{2}$. One obtains for $k \geq k_{2}$ : 


$$
\begin{aligned}
& \int_{K}\left[\phi(x)-f_{l_{k}}\left(x, v_{l_{k}}(x)\right)\right] \mathrm{d} x \\
= & \int_{K}\left[\phi(x)-f^{+}(x, 0)\right] \mathrm{d} x+\int_{K}\left[f^{+}(x, 0)-f_{l_{k}}\left(x, v_{l_{k}}(x)\right)\right] \mathrm{d} x \\
= & \varepsilon+\int_{K}\left[f^{+}(x, 0)-f^{+}\left(x, v_{l_{k}}(x)\right)\right] \mathrm{d} x \\
\geq & \frac{\varepsilon}{2},
\end{aligned}
$$

which contradicts $f_{l_{k}}\left(\cdot, v_{l_{k}}(\cdot)\right) \rightarrow \phi$. Thus, meas $(K)=0$.

Now, let $A$ be the closed linear operator in $L^{2}(0,1)$ defined by

$$
\operatorname{dom}(A):=\left\{\varphi \in W^{2,2}[0,1]: \varphi^{\prime}(0)=0=\varphi^{\prime}(1)\right\}
$$

and $A \phi:=-\phi "+q \phi$. Clearly,

$$
f_{l_{k}}\left(x, v_{l_{k}}(x)\right) \rightarrow \phi(x),
$$

hence $v_{l} \rightarrow u$ and the fact that $A$ is weakly closed yields

$$
A u=\lambda \phi,
$$

i.e.

$$
A u \in \lambda F(\cdot, u(\cdot)) \text { a.e. }
$$

Finally, we show that $u \in W^{2, \infty}(0,1)$. In fact, from (3.9) we have

$$
u^{\prime \prime}(x)=q(x) u(x)-\lambda \phi(x) .
$$

According to (H1) and the boundedness of $u$ we have

$$
q u \in L^{\infty}(0,1) .
$$

We claim that $\varphi \in L^{\infty}(0,1)$. Suppose on the contrary that there exists a set $E \subset[0,1]$, meas $(E)>0$ such that $|\varphi|$ is unbounded on $E$. Without loss of generality, we assume that

$$
|\phi(s)|>\frac{M\|w\|_{L^{2}}+1-\int_{[0,1] \backslash E}|\phi(x)||w(x)| \mathrm{d} x}{\int_{E}|w(x)| \mathrm{d} x}, \quad s \in E,
$$

where $M$ is given by $(3.7)$ and $w \in L^{2}(0,1)$. On the one hand, for $k$ larger enough from (3.7), (3.8) and (H2) we have

$$
\int_{0}^{1}|\phi(x)||w(x)| \mathrm{d} x \leq \int_{0}^{1}\left|f_{l_{k}}\left(x, v_{l_{k}}(x)\right) w(x)\right| \mathrm{d} x+1 \leq M\|w\|_{L^{2}}+1 .
$$

On the other hand, from (3.12) we have

$$
\begin{aligned}
& \int_{0}^{1}|\phi(x)||w(x)| \mathrm{d} x \\
= & \int_{E}|\phi(x)||w(x)| \mathrm{d} x+\int_{[0,1] \backslash E}|\phi(x)||w(x)| \mathrm{d} x \\
> & \frac{M\|v\|_{L^{2}}+1-\int_{[0,1] E E}|\phi(x)||w(x)| \mathrm{d} x}{\int_{E}|w(x)| \mathrm{d} x} \int_{E}|w(x)| \mathrm{d} x+\int_{[0,1] \backslash E}|\phi(x)||w(x)| \mathrm{d} x \\
= & M\|w\|_{L^{2}}+1,
\end{aligned}
$$


which contradicts (3.13). Thus,

$$
\phi \in L^{\infty}(0,1) .
$$

Therefore, from (3.10), (3.11) and (3.14) we obtain $u \in W^{2, \infty}(0,1)$.

Now we are in the position to prove Theorem 3.1.

\section{Proof of Theorem 3.1.}

Assume that $(\lambda, u) \in C_{1}^{+}$. We divide the proof into two cases.

Case 1. If $\|u\|_{\infty}=\xi_{2 j-1}$ for some $j \in \mathbb{N}^{*}$, then $\lambda \geq 2$.

Since $(\lambda, u) \in C_{1}^{+}$, there exists a sequence $\left(\lambda_{k_{i}}, z_{k_{i}}\right) \in C_{1, k_{i}}^{+}$, such that

$$
\lim _{i \rightarrow \infty} \lambda_{k_{i}}=\lambda, \lim _{i \rightarrow \infty} z_{k_{i}}=u .
$$

Hence, for $\delta>0$ there exists $i_{0} \in \mathbb{N}$, such that

$$
\left\|z_{k_{i}}-u\right\|_{\infty}<\delta, \quad i>i_{0}
$$

i.e.

$$
\xi_{2 j-1}-\delta<\left\|z_{k_{i}}\right\|_{\infty}<\xi_{2 j-1}+\delta, \quad i>i_{0} .
$$

By using Lemma 3.4, we obtain that

$$
\lambda_{k_{i}}>2, \quad i>i_{0} .
$$

Hence, we get

$$
\lambda=\lim _{i \rightarrow \infty} \lambda_{k_{i}} \geq 2 .
$$

Case 2. If $\|u\|_{\infty}=\xi_{2 j}$ for some $j \in \mathbb{N}^{*}$, then $\lambda \leq \frac{1}{2}$.

Since $(\lambda, u) \in C_{1}^{+}$, there exists a sequence $\left(\lambda_{k_{i}}, z_{k_{i}}\right) \in C_{1, k_{i}}^{+}$, such that

$$
\lim _{i \rightarrow \infty} \lambda_{k_{i}}=\lambda, \quad \lim _{i \rightarrow \infty} z_{k_{i}}=u
$$

Hence, for $\delta>0$ there exists $i_{0} \in \mathbb{N}$, such that

$$
\left\|z_{k_{i}}-u\right\|_{\infty}<\delta, \quad i>i_{0}
$$

i.e.

$$
\xi_{2 j}-\delta<\left\|z_{k_{i}}\right\|_{\infty}<\xi_{2 j}+\delta, \quad i>i_{0} .
$$

By using lemma 3.4, we obtain that

$$
\lambda_{k_{i}}<\frac{1}{2}, \quad i>i_{0}
$$

Hence, we get

$$
\lambda=\lim _{i \rightarrow \infty} \lambda_{k_{i}} \leq \frac{1}{2} .
$$

Corollary 3.1. Assume that (H1)-(H3) hold. Then

(i) for each $\lambda \in\left(0, \frac{1}{2}\right),(1.1)$ has at least one positive solution: $u_{0} \in C_{1}^{+}$; 
(ii) for each $\lambda \in\left[\frac{1}{2}, 2\right]$, (1.1) has $N$ positive solutions:

$$
u_{k}, k=1,2, \ldots, N \text {, }
$$

which satisfy that $u_{k} \in C_{1}^{+}, k=1,2, \ldots, N$.

Proof. According to Theorem 3.1, the boundary value problem (1.1) has an unbounded component $C_{1}^{+}$in $\Sigma$ with $(0,0) \in C_{1}^{+}$. Moreover,

(i) $(\lambda, u) \in C_{1}^{+}$with $\|u\|_{\infty}=\xi_{2 j-1}$ for some $j \in \mathbb{N}^{*}$ implies that $\lambda \geq 2$;

( $\widetilde{i i})(\lambda, u) \in C_{1}^{+}$with $\|u\|_{\infty}=\xi_{2 j}$ for some $j \in \mathbb{N}^{*}$ implies that $\lambda \leq \frac{1}{2}$.

From the facts $(0,0) \in C_{1}^{+}$, and $(\lambda, u) \in C_{1}^{+}$with $\|u\|_{\infty}=\xi_{1}$ implies that $\lambda \geq 2$ and the connectivity of $C_{1}^{+}$, we obtain

$$
C_{1}^{+} \cap \lambda \times C^{1}[0,1] \neq \emptyset, \quad \forall \lambda \in\left(0, \frac{1}{2}\right),
$$

which implies for each $\lambda \in\left(0, \frac{1}{2}\right),(1.1)$ has at least one positive solution: $u_{0} \in C_{1}^{+}$.

Let

$$
C_{1}^{+, k}:=\left\{(\lambda, u) \in C_{1}^{+} \mid \xi_{k-1} \leq\|u\|<\xi_{k}\right\}, \quad k=1,2, \ldots, N,
$$

where $\xi_{0}=0, \xi_{k}(k=1,2, \ldots, N)$ is given by (H3). Then according to $(\tilde{i})(\tilde{i})$ and the connectivity of $C_{1}^{+}$, we obtain

$$
C_{1}^{+, k} \cap \lambda \times C^{1}[0,1] \neq \emptyset, \quad \forall \lambda \in\left(\frac{1}{2}, 2\right), \quad k=1,2, \ldots, N,
$$

which implies for each $\lambda \in\left(\frac{1}{2}, 2\right)$, (1.1) has $N$ positive solutions:

$$
u_{k}, k=1,2, \ldots, N \text {, }
$$

and $u_{k} \in C_{1}^{+, k} \subset C_{1}^{+}, k=1,2, \ldots, N$.

\section{Example}

In this section, an example is given to illustrate the application of our main result (Theorem 3.1). Consider second order Neumann differential inclusion problem

$$
\left\{\begin{array}{l}
-u^{\prime \prime}(x)+u(x) \in \lambda F(u(x)), \quad \text { a.e. } x \in(0,1), \\
u^{\prime}(0)=0, \quad u^{\prime}(1)=0,
\end{array}\right.
$$

where the set-valued function $F$ in (4.1) is given by

$$
F(\gamma)=\left\{\begin{array}{l}
\frac{49}{2} y-\frac{195}{2}, \quad y \geq 4 \\
\frac{1}{16} y+\frac{1}{4}, \quad 0<y<4 \\
{\left[0, \frac{1}{4}\right], \quad y=0 .}
\end{array}\right.
$$

Obviously, (H1), (H2) conditions of Theorem 3.1 are satisfied. Moreover, Green's function of the associated linear problem

$$
\left\{\begin{array}{l}
-u^{\prime \prime}+u=0, \\
u^{\prime}(0)=0, \quad u^{\prime}(1)=0,
\end{array}\right.
$$


can be explicitly expressed by

$$
G(x, s)=\frac{1}{2\left(e-e^{-1}\right)}\left\{\begin{array}{l}
\left(e^{x-1}+e^{1-x}\right)\left(e^{s}+e^{-s}\right), 0 \leq s \leq x \leq 1 \\
\left(e^{x}+e^{-x}\right)\left(e^{s-1}+e^{1-s}\right), 0 \leq x \leq s \leq 1 .
\end{array}\right.
$$

By calculation we can get $\int_{0}^{1} G(s, s) \mathrm{d} s=\frac{e}{e-e^{-1}}, \int_{0}^{1} G\left(\frac{1}{2}, s\right) \mathrm{d} s=1$ and $\sigma=\frac{2}{e+e^{-1}}$.

Let $\xi_{1}=3, \xi_{2}=11, \delta=1$, then we can check that $\xi_{1}=3<5<\sigma\left(\xi_{2}-\delta\right)$, and

$$
\begin{aligned}
& \Phi\left(\xi_{1}\right)=\frac{1}{2}<\frac{3}{4}<1-e^{-2}=\frac{1}{2}\left(\int_{0}^{1} G(s, s) \mathrm{d} s\right)^{-1}\left(\xi_{1}-\delta\right), \\
& \Psi\left(\xi_{2}\right) \geq 25>24=2\left(\int_{0}^{1} G\left(\frac{1}{2}, s\right) \mathrm{d} s\right)^{-1}\left(\xi_{2}+\delta\right) .
\end{aligned}
$$

So that $(H 3)$ condition of Theorem 3.1 is satisfied. Therefore, according to Theorem 3.1 the differential inclusion problem (4.1) has an unbounded component $C_{1}^{+}$in $\Sigma$ with $(0,0) \in C_{1}^{+}$. Moreover,

(i) $(\lambda, u) \in C_{1}^{+}$with $\|u\|_{\infty}=3$ implies that $\lambda \geq 2$;

(ii) $(\lambda, u) \in C_{1}^{+}$with $\|u\|_{\infty}=11$ implies that $\lambda \leq \frac{1}{2}$.

\section{Acknowledgements}

The authors express their gratitude to Professors Ma Tian and Ma Ruyun for their guidance and encouragement, also to an anonymous referee for a number of valuable comments and suggestions.

\section{Competing interests}

The authors declare that they have no competing interests.

Received: 14 October 2011 Accepted: 23 April 2012 Published: 23 April 2012

\section{References}

1. Aubin, JP, Cellina, A: Differential Inclusion, vol. 264. Grundlehren Math Wiss. Springer-Verlag, Berlin (1984)

2. Kunze, M: Nonsmooth dynamical systems. Lecture Notes in Mathematics, vol. 1744. Springer-Verlag, Berlin (2000)

3. Clarke, FH, Ldeyaev, YS, Stern, RJ, Wolenski, PR: Nonsmooth Analysis and Control Theory. Springer-Verlag, New York (1998)

4. Clarke, FH: Optimization and Nonsmooth Analysis. SIAM, Philadelphia (1990)

5. Leine, Rl, Nijjmeijer, H: Dynamics and bifurcation of nonsmooth mechanical systems. Lecture Notes in Applied and Computational Mechanics, vol. 18. Springer-Verlag, Berlin (2004)

6. Gasiésdi, L, Papageorgion, NS: Nonsmooth Critical Point Theory and Nonlinear Boundary Value Problems. Chapman and Hall/CRC, Boca Raton (2005)

7. Deimling, K: Multivalued Differential Equation. Springer-Verlag, Berlin (1985)

8. Kowalczyk, P, Piiroinen, PT: Two-parameter sliding bifurcation of periodic solutions in a dry-friction oscillator. Physica D. 237, 1053-1073 (2008). doi:10.1016/j.physd.2007.12.007

9. $\quad$ Deimling, K: Resonance and clulomb friction. Diff Integr Equ. 7(3):759-765 (1994)

10. Ma, R: Existence of periodic solutions of a generalized friction oscillator. Nonlinear Anal Real World Appl. 11, 3316-3322 (2010). doi:10.1016/j.nonrwa.2009.11.024

11. Chang, KC: Variational methods for nondifferentable functionals and their applications to partial differential equations. J Math Anal Appl. 80, 102-112 (1981). doi:10.1016/0022-247X(81)90095-0

12. Kourogenis, NC, Papageorgiou, NS: Nonsmooth critical point theory and nonlinear elliptic equation at resonance. Kodai Math J. 23, 128-135 (2000)

13. Zykov, PS: On two-point boundary value problems for second-order differential inclusions on manifolds. Appl Anal. 88(6):895-902 (2009). doi:10.1080/00036810903042232

14. Hannelore, L, Csaba, V: Multiple solutions for a differential inclusion problem with nonhomogeneous boundary conditions. Numer Funct Anal Optim. 30(5-6):566-581 (2009). doi:10.1080/01630560902987857

15. Ntouyas, SK, O' Regan, D: Existence results for semilinear neutral differential inclusions with nonlocal conditions. Diff Equ Appl. 1(1):41-65 (2009)

16. Papageorgion, NS, Staicu, V: The method of upper-lower solutions for nonlinear second order differential inclusions. Nonlinear Anal. 67(3):708-726 (2007). doi:10.1016/j.na.2006.06.023 
17. Kyritsi, S, Matzakos, N, Papageorgion, NS: Periodic problems for strongly nonlinear second-order differential inclusions. J Diff Equ. 183, 279-302 (1982)

18. Dhage, BC, Ntouyas, SK, Cho, YJ: On the second order discontinuous differential inclusions. J Appl Funt Anal. 1(4):469-476 (2006)

19. Benchchra, M, Graef, JR, Ouahab, A: Oscillatory and nonoscillatory solutions of multivalued differential inclusions. Comput Math Appl. 49(9-10):1347-1354 (2005). doi:10.1016/j.camwa.2004.12.007

20. Budyko, Ml: The effect of solar radiation variations on the climate of the earth. Tellus. 21, 611-619 (1969). doi:10.1111/ j.2153-3490.1969.tb00466.x

21. Diaz, Jl: Mathematical Analysis of Some Diffusive Energy Balance Models. Math Climate Environ. Mason, Paris (1993)

22. Diaz, J: The Mathematics of Models for Climatology and Environment, NATO ASI Series I: Global Environmental Changes, vol. 48. Springer-Verlag, New York (1997)

23. Diaz, Jl, Hernandez, J, Tello, L: On the multiplicity of equilibrium solutions to a nonlinear diffusion equation on a manifold arising in climatology. J Math Anal Appl. 216, 593-613 (1997). doi:10.1006/jmaa.1997.5691

24. Henderson-Sellers, A, McGuffie, KA: A Climate Modeling Primer. Wiley, Chich-ester (1987)

25. Hetzer, G: A bifurcation result for Sturm-Liouville problem with a set-valued term. Mississippi State University (1997) In Proceedings of the Third Mississippi State Conference on Difference Equations and Computational Simulations: 16-17 May 1997

26. Jiang, D, Liu, H: Existence of positive solutions to second order Neumann boundary value problem. J Math Res Expo. 20, 360-364 (2000)

27. Chu, JF, Sun, YG, Chen, H: Positive solutions of Neumann problems with singularities. J Math Anal Appl. 337, 1267-1272 (2008). doi:10.1016/j.jmaa.2007.04.070

28. Sun, Y, Cho, YJ, O' Regan, D: Positive solution for singular second order Neumann boundary value problems via a cone fixed point theorem. Appl Math Comput. 210, 80-86 (2009). doi:10.1016/j.amc.2008.11.025

29. Whyburn, GT: Topological Analysis. Princeton University Press, Princeton, NJ (1964)

30. $\mathrm{Ma}, \mathrm{R}, \mathrm{An}, \mathrm{Y}$ : Global structure of positive solutions for nonlocal boundary value problems involving integral conditions. Nonlinear Anal TMA. 71(10):4364-4376 (2009). doi:10.1016/j.na.2009.02.113

31. Kuratowski, C: Topologie II. Warszawa (1950)

32. Zeidler, E: Nonlinear Functional Analysis and its Applications I (Fixed-point theorems). Springer-Verlag, New York (1986)

33. Mavinga, N, Nkashama, MN: Steklov-Neumann eigenproblems and nonlinear elliptic equations with nonlinear boundary conditions. J Diff Equ. 248, 1212-1229 (2010). doi:10.1016/j.jde.2009.10.005

doi:10.1186/1687-2770-2012-47

Cite this article as: Yan: Oscillating global continua of positive solutions of second order Neumann problem with a set-valued term. Boundary Value Problems 2012 2012:47.

\section{Submit your manuscript to a SpringerOpen ${ }^{\odot}$} journal and benefit from:

- Convenient online submission

- Rigorous peer review

- Immediate publication on acceptance

- Open access: articles freely available online

- High visibility within the field

- Retaining the copyright to your article

Submit your next manuscript at $\gg$ springeropen.com 